[12] W. Sierpiński, Sur les images biunivoques et continues de l'ensemble de tous les nombres irrationnels, Mathematica 1 (1929), pp. 18-21.

[13] J. M. Smirnov, Oontractions to bicompacta and their connection with retraction and bicompact extensions, Fund. Math. 63 (1968), pp. 199-211.

[14] G. T. Whyburn, Analytic Topology, Amer. Math. Soc., Providence, R. I., 1942

UNIVERSITY OF NORTH CAROLINA AT CHARLOTTE

Charlotte, North Carolina

Accepté par la Ródaction le 28, 12. 19\%3

\section{Infinite dimensional non-symmetric Borsuk-Ulam theorem}

by

Kapill D. Joshi (Providence, Rhode Island)

Abstract. Let $R^{\infty}$ be an infinite dimensional Banach space and $R^{\infty-1}$ a closed subspace of codimension one. If $X \subset R^{\infty}$, a mapping $f: X \rightarrow R^{\infty}$ is said to be a compact vector field or a compact field if the associated displacement mapping $F: X \rightarrow R^{\infty}$ defined by $F(x)=x-f(x)$ maps $X$ into a compact subset of $R^{\infty}$. It is proved that if $X$ is closed and bounded and if the origin lios in a bounded component of the compleme $R^{\infty}$ : then for any compact field $f: X \rightarrow R^{\infty-1}$ there exist two points $x$ and $y$ in $X$, Iying on opposite rays from the origin (i.e. $y=-\lambda x$ for some $\lambda>0$ ), such that $f(x)=f(y)$. This is a generalization of a theorem of Granas which results by taking $X$ to be the unit sphere in $R^{\infty}$. The proof uses techniques analogous to those of Granas to reduce the problem to the finite dimensional case which was proved earlier by the author.

1. Introduction. The classic Borsuk-Ulam theorem states that if $f: S^{n} \rightarrow R^{n}$ is a map of the $n$-sphere into the Euclidean space $R^{n}$ then there exists a pair of antipodal points $\{x,-x\}$ on $S^{n}$ such that $f(x)=f(-x)$. Several generalizations of this theorem, preceeding in various directions, are known (see, for example, the references in [2]). In some of these generalizations the sphere is replaced by a more general space on which some suitable notaion of antipodality can be defined. In particular, the author [2] has proved the following theorem conjectured by Borsuk.

THworem A. Let $X$ be a compact subset of the Euclidean space $R^{n+1}$ which disconnects it in such a way that the origin lies in a bounded component of $R^{n+1}-X$. Then for any map $f: X \rightarrow R^{n}$ there exist two points $x$ and $y$ in $X$, Tying on opposite rays from the origin (that is, $y=-\lambda x$ for some $\lambda>0$ ), such that $f(x)=f(y)$.

On the other hand Granas [1] has extended the Borsuk-Ulam theorem from Euclidean spaces to infinite dimensional Banach spaces. Let $R^{\infty}$ denote a fixed infinite dimensional Banach space and $S^{\infty}$ the unit sphere in $R^{\infty}$. By $R^{\infty-1}$ we mean a linear, closed subspace of $R^{\infty}$ of co-dimension one. Because the unit sphere $S^{\infty}$ is not compact, the BorsukUlam theorem does not hold for an arbitrary map $f: S^{\infty} \rightarrow R^{\infty-1}$. However if the mapping $f$ does not displace points of $S^{\infty}$ too much (i.e. if the mapp- 
ing $F: S^{\infty} \rightarrow R^{\infty}$ defined by $F(x)=x-f(x)$ is in some sense "small") then the Borsuk-Ulam theorem holds for $f$. Granas [1] uses the term "compact fields" for such mappings and the precise definition will be given later. Granas' theorem can now be stated as follows.

THEOREM B. Let $f: S^{\infty} \rightarrow R^{\infty-1}$ be a mapping of the unit sphere $S^{\infty}$ into a 1-codimensional subspace $R^{\infty-1}$ of $R^{\infty}$. If $f$ is a compact field then $f$ maps at least one pair of antipodal points into the same point, i.e. $f(x)=f(-\infty)$ for some $x \in S^{\infty}$.

(See [1], p. 45.)

The purpose of this paper is to combine Theorems A and $B$. Specifically our theorem is,

THeOREM C. Let $X$ be a closed, bounded subset of $R^{\infty}$ for which the origin is in a bounded component of $R^{\infty}-X$ and let $f: X \rightarrow R^{\infty-1}$ be $a$ compact field. Then there exist two points $x$ and $y$ in $X$ and a positive real number $\lambda$, such that $y=-\lambda x$ and $f(x)=f(y)$.

On one hand Theorem $O$ can be regarded as a generalization of Theorem $\mathrm{B}$ the way Theorem $\mathrm{A}$ is a generalization of the Borsuk-Ulam theorem. On the other hand it can also be considered as an infinite dimensional extension of Theorem $A$ the way Theorem $B$ is an extension of the Borsuk-Ulam theorem. These two points of view suggest two corresponding lines of approach to Theorem $O$. Thus one may try to prove it using the same techniques as those used in the proof of Theorem A as given in [2]. Unfortunately this approach does not seem to work. For, the proof in [2] is based on the theory of the Smith index of an involution and the technique of approximating the set $X$ by a suitable type of compact polyhedra. Both of these rely crucially on compactness of the set $X$ in Theorem $\mathrm{A}$ and it is doubtful if either of these can be readily extended to the non-compact case as would be required by Theorem $\mathrm{C}$.

We therefore try the other approach which is similar to the one used by Granas [1] in proving Theorem B. Here the idea is to approximate the map $f: X \rightarrow R^{\infty-1}$ by finite dimensional maps to which Theorem A may be applied. Even this approach presents some difficulties; but these are technical and can be overcome by using sharper versions of the theorems used by Granas.

In the second section we review and extend some of the results rsed by Granas. In the last section we apply these together with Theorem A to prove Theorem $\mathrm{C}$.

2. Compact vector fields and their approximation. In this section we recall the definition and some of the properties of compact vector fields. We shall also prove an approximation theorem analogous to the one in ([1], p. 24). For details the reader is referred to [1]. The properties of
Banach spaces which we shall use are all very standard and can be found for example in [3].

Let $I$ denote a fixed real Banach space (that is, a complete, normed, linear space which is not necessarily separable). By a subspace we always mean a closed linear subspace.

(2.1) Defintrion. Let $X$ be a metric space. A mapping $F: X \rightarrow E$ is said to be compact if its range $F(X)$ is contained in a compact subset of $E$; that is if $\overline{F^{\prime}(\bar{X})}$ is compact.

(2.2) Defrntron. A mapping $T^{\prime}: X \rightarrow E$ is said to be finite dimensional if $F$ is compact and $F^{\prime}(X)$ is contained in some finite dimensional subspace $E^{n}$ of $E$.

The proof of the following proposition is trivial.

(2.3) Propostrion. Let $E, E^{\prime}$ be Banach spaces and let $\pi: E \rightarrow E^{\prime}$ be linear and continuous. Then if $F: X \rightarrow E$ is compact (or finite dimensional), so is the composite $\pi \circ \mathbb{H}: X \rightarrow \mathbb{W}^{\prime}$.

The following theorem asserts that a compact mapping can be uniformly approximated by finite dimensional mappings. A proof is given by Granas ([1], p. 24).

(2.4) THeorem. If $F: X \rightarrow E$ is a compact mapping then for every $\varepsilon>0$ there exists a finite dimensional mapping $F_{\mathrm{g}}: X \rightarrow \mathbb{E}^{n}$ such that $\left\|F(x)-F_{s}(x)\right\|<\varepsilon$ for every $x \in X$. (Here \|\| denotes the norm on $\mathbb{E}$ and the integer $n$ may of course depend on $\varepsilon$ ).

We shall need a sharper version of the preceding theorem in that the approximating map $F_{s}$ will have to satisfy some additional property besides being finite dimensional. Before stating it we introduce some notation. Suppose the space $E$ is the direct sum of two subspaces, $A$ and $B$ i.e. $E=A \oplus B$. Then every element of $E$ can be written uniquely as $a+b$ where $a \in A$ and $b \in B$. Note that since $A$ and $B$ are closed they are Banach spaces themselves. Combining this fact with the well-known "Closed Graph Theorem" (seo [3], p. 196) it follows that the projection maps $\pi_{A}: D \rightarrow A$ and $\pi_{B}: Z \rightarrow B$ defined by $\pi_{A}(a+b)=a$ and $\pi_{B}(a+b)=b$ are continuous. Obviously they are also linear. The dimension of $B$ is called the codimension of $A$ in $D$. This is well-defined since it coincides with the dimension of the quotient space $E / A$.

We are interested in the case in which $A$ is of co-dimension one. In this case $A$ can be regarded as the kernel of a continuous linear functional $L$ on $E$. Indeed, since $E=A \oplus B$ where $B$ is one-dimensional, we morely take any continuous, linear isomorphism $\theta: B \rightarrow R$ and set $L=\theta \circ \pi_{B}$. Conversely it is clear that the kernel of a non-zero continuous linear functional on $E$ is a subspace of codimension 1 .

Returning to the approximation theorem suppose a compact map $F: X \rightarrow E$ and a continuous linear functional $L: E \rightarrow R$ are given. If we 
think of $I$ as a "level function" on $I$ then we claim that the approximating map $F_{s}$ in (2.4) can be chosen to be "on the same level" as the map $F$. The precise statement is:

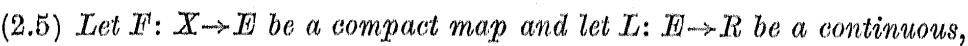
linear functional. Then for every $\varepsilon>0$ there exists a finite dimensional mapping $\mathbb{F}_{\varepsilon}: X \rightarrow H^{\text {to }}$ such that $\left\|F^{\prime}(x)-W_{a}(x)\right\|<\varepsilon$ and $L\left(F^{\prime}(x)\right)=L\left(F_{a}(x)\right)$ for every $x \in X$.

Proof. If $L$ is identically zero then the result follows from (2.4) immediately. If $L$ is non-zero let $A$ be its kernel. As remarked above, there is a 1 -dimensional subspace $B$ of $t$ such that $D=A \oplus B$. If we identify $B$ and $R$ then $L$ is just the projection $\pi_{B}: E \rightarrow B$. We let $G: X \rightarrow A$ be the composite $\pi_{A} \circ F$. Since $\pi_{A}$ is continuous, by (2.3) $G$ is a compact mapping of $X$ into the Banach space $A$. Hence by (2.4) there exists a finite dimensional subspace $E^{n}$ of $A$ and a finite dimensional mapping $G_{s}: X \rightarrow \mathbb{R}^{n}$ such that $\left\|G(x)-G_{g}(x)\right\|<\varepsilon$ for every $x \in X$. We let $k=n+1$ and $E^{t c}$ be the subspace of $E$ spanned by $E^{n}$ and $B$. Define $T_{z}: X \rightarrow \mathbb{E}^{h}$ by $F_{s}(x)=G_{s}(x)+\pi_{B}\left(T^{\prime}(x)\right)$ for $x \in X$. Obviously for every $x \in X, I(x)-$ $-F_{s}(x)=G(x)-G_{s}(x)$ since $F(x)=\pi_{A}\left(F^{\prime}(x)\right)+\pi_{B}\left(F^{\prime}(x)\right)$. Hence,

$$
\left\|F^{\prime}(x)-F_{B}(x)\right\|<\varepsilon .
$$

It is also clear that $L\left(F^{\prime}(x)\right)=L\left(F_{\theta}(x)\right)$ since $L$ coincides with $\pi_{B}$. To complete the proof it only remains to show that $F_{\text {s }}$ is compact. For this we note that if $S$ and $T$ are two compact sets of a Banach space then the set $S+T$ defined as the set $\{(s+t) \mid s \in S, t \in T\}$ is also compact by continuity of the addition operation in $\mathbb{E}$. In the present case we take $S$ $=\overline{G_{\varepsilon}(X)}$ and $T=\pi_{B} \overline{(F(X))}$. Then $S$ and $T$ are compact because the mappings $G_{s}$ and $F$ are compact. Clearly $F_{s}$ maps $X$ into the set $S+T$ which is compact. This completes the proof.

So far the space $X$ was assumed to be any metric space. Suppose now that $X$ itself is a subset of $E$. Then for each $x \in X$ we can consider the "displacement" $x-F(x)$ where $F: X \rightarrow E$ is a given mapping.

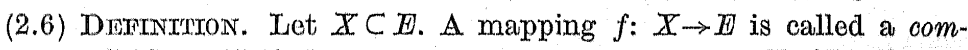
pact vector field on $X$ if there exists a compact mapping $F: X \rightarrow D$ such that $f(x)=x-I F(x)$ for each $x \in X$. (Clearly $F$ is uniquely determined by $f$.)

We conclude this section with a theorem which is an easy consequence of (2.5) and which will be used in the next section in the proof of Theorem $\mathrm{O}$ in order to reduce the problem to finite dimensional spaces.

(2.7) THEorim. Suppose a compact vector field $f: X \rightarrow E$ maps $X$ into a 1-codimensional subspace $A$ of $\mathbb{E}$. Then for every $\varepsilon>0$ there exists a finite dimensional subspace $\mathbb{E}^{k}$ of $\mathbb{E}$ and a compact vector field $f_{s}: X \rightarrow E$ such that for each $x \in X,\left\|f(x)-f_{\varepsilon}(x)\right\|<\varepsilon$ and $f_{s}$ maps $X \cap \mathbb{E}^{k}$ into a 1 -codimensional subspace $\mathbb{E}^{k-1}$ of $\mathbb{B}^{k}$.
Pro of. We regard $A$ as the kernel of a continuous linear functional $L$ on $E$. The rest of the notation is as in the proof of (2.5). Let $f(x)=x-$ $-F(x)$ where $F: X \rightarrow E$ is a compact mapping. The hypothesis implies that $L(x)=L(F(x))$ for every $x \in X$. Let $E^{k}$ and the compact mapping $F_{\varepsilon}: X \rightarrow E^{k}$ be as given by (2.5). We define the compact vector field $f_{\varepsilon}: X \rightarrow E$ by $f(x)=x-F_{\varepsilon}(x)$ for $x \in X$. Clearly for every $x \in X, f(x)-f_{s}(x)$ $=F^{\prime}(x)-F_{\varepsilon}(x)$ and hence $\left\|f(x)-f_{s}(x)\right\|<\varepsilon$. Also $L\left(F_{s}(x)\right)=L(F(x))$ and hence $L\left(f_{\varepsilon}(x)\right)=0$ for each $x \in X$. This means that $f_{s}$ maps $X$ into $A$. Now if $x \in X \cap E^{k}$ then $f_{e}(x) \in E^{k}$. Thus $f_{s}$ maps $X \cap E^{k}$ into $E^{k} \cap A$. The latter is clearly a $(k-1)$-dimensional subspace of $E$ and so the proof is complete.

3. The main result. In this section we combine Theorem $A$ in the introduction with Theorem (2.7) in the last section to prove the desired infinite dimensional, non-symmetric Borsuk-Ulam theorem.

We shall consider a fixed infinite dimensional Banach space which we shall denote by $R^{\infty}$ rather than by $E$ as was done in the last section. Its finite dimensional subspaces will be denoted by $R^{n}$ etc. and by $R^{\infty-1}$ we mean a fixed 1-codimensional subspace of $R^{\infty}$. Let $X$ be a closed and bounded subset of $R^{\infty}$ which disconnects it in such a way that the origin lies in a bounded component of the complement $R^{\infty}-X$. If $R^{n}$ is a subspace of $R^{\infty}$ of finite dimension then $X \cap R^{n}$ is a compact subset of $R^{n}$ and the origin (in $R^{n}$ ) clearly lies in a bounded component of the complement of $X \cap R^{n}$ in $R^{n}$. This fact will be crucial in the proof. First we establish a lemma.

(3.1) LEMIVA. Let $X$ be as above and let $f: X \rightarrow R^{\infty}$ be a compact vector field. If for every $\delta>0$ there exist points $x,-\lambda x$ in $X$ with $\lambda>0$ for which $\|f(x)-f(-\lambda x)\|<\delta$ then there exist such points for which $f(x)=f(-\lambda x)$.

Proof. The fact that $X$ is closed, bounded and does not contain the origin implies the existence of two positive real numbers $r$ and $R$ such that for every $x \in X, r \leqslant\|x\| \leqslant R$. It follows that if for some $\lambda>0, x$ and $-\lambda x$ are both in $X$ then $r / R \leqslant \lambda \leqslant R / r$. Taking $\delta=1, \frac{1}{2}, \frac{1}{3}, \frac{1}{4}, \ldots$ succesively we get a sequence $\left\{x_{i}\right\}$ of points in $X$ and a sequence $\left\{\lambda_{i}\right\}$ of positive real numbers such that for each $i,-\lambda_{i} x_{i} \in X$ and $\left\|f\left(x_{i}\right)-f\left(-\lambda_{i} x_{i}\right)\right\|<1 / i$. If the set $X$ were compact then we could have taken convergent subsequences to reach the conclusion of the lemma. Unfortunately $X$ cannot be compact. However we can write $f(x)=x-F(x)$ for each $x \in X$ where the mapping $F: X \rightarrow R^{\infty}$ is compact. This means that the set $\overline{F(X)}$ is compact. Also each $\lambda_{i}$ is in the closed interval $[r / R, R / r]$ which is compact. Thus taking suitable subsequences if necessary we may assume that the sequences $\left\{F\left(x_{i}\right)\right\},\left\{\lambda_{i}\right\}$ and $\left\{F\left(-\lambda_{i} x_{i}\right)\right\}$ are all convergent. Let $y_{i}=f\left(x_{i}\right)$ $-f\left(-\lambda_{i} x_{i}\right)$ for each $i$. Then the sequence $\left\{y_{i}\right\}$ also converges; in fact it converges to 0 by its construction. An easy computation shows that for 4 - Fundamenta Mathematicae LXXXIX 
each $i$,

$$
x_{i}=\frac{F\left(x_{i}\right)-F\left(-\lambda_{i} x_{i}\right)-y_{i}}{1+\lambda_{i}} .
$$

From (*) it follows that the sequence $x_{i}$ is also convergent. Once this is established the lemma follows by a routine argument using continuity of $f$ and the fact that the set $X$ is closed.

We are now in a position to prove the main result.

(3.2) THEOREM. Let $X$ be a closed, bounded subset of $R^{\infty}$ for which the origin is in a bounded component of $R^{\infty}-X$ and let $f: X \rightarrow R^{\infty-1}$ be a compact vector field. Then there exist two points $x$ and $y$ in $X$ and a positive real number $\lambda$, such that $y=-\lambda x$ and $f(x)=f(y)$.

Proof. In view of the preceding lemma it suffices to find such $x$, $y, \lambda$ so that $\|f(x)-f(y)\|<\delta$ where $\delta$ is a pre-assigned positive number. Given such $\delta$ we set $\varepsilon=\frac{1}{2} \delta$ and apply (2.7). This gives a finite dimensional subspace $R^{k}$ of $R^{\infty}$ and a compact vector field $f_{8}: X \rightarrow R^{\infty}$ such that $f_{8}$ maps $X \cap R^{k}$ into a $(k-1)$-dimensional subspace $R^{k-1}$ and $\left\|f(x)-f_{s}(x)\right\|$ $<\frac{1}{2} \delta$ for every $x \in X$. As observed earlier, the set $X \cap R^{k}$ is a compact subset of $R^{k}$ for which the origin lies in a bounded component of $R^{k}$ $-\left(X \cap R^{k}\right)$. Applying Theorem $\mathrm{A}$ in the introduction to the restriction map $f_{s}: X \cap R^{k} \rightarrow R^{k-1}$ we get points $x$ and $y$ in $X \cap R^{k}$ and $\lambda>0$ such that $f_{s}(x)=f_{\varepsilon}(y)$ and $y=-\lambda x$. Since we have, $\|f(x)-f(y)\| \leqslant \| f(x)$ $-f_{\varepsilon}(x)\|+\| f_{8}(x)-f_{\varepsilon}(y)\|+\| f_{B}(y)-f(y) \|$, it follows that $\|f(x)-f(y)\|<\delta$ and the theorem is proved.

\section{References}

[1] A. Granas, The theory of compact vector fields and some of its applications to topology of functional spaces (I), Dissertationes Math. 30 (1962).

2] K. D. Joshi, A non-symmetric generalization of the Borsuk-Ulam theorem, Fund. Math. 80 (1973), pp. 13-33.

[3] H. L. Royden, Real Analysis, New York 1968.

\section{DEPARTMENT OF MATHEMATICS}

BROWN UNIVERSITY

Providence, Rhode Island

DEPARTMENT OF MATHEMATICS

INDIAN INSTITUTE OF TECHNOLOGY

Bombay, India

\section{Wide tree-like spaces have a fixed point}

by

John Jobe (Stillwater, Okla.)

Abstract. A well-known unsolved problem is to determine whether or not a compact plane continuum which does not separate the plane has the fixed point property for continuous functions. In this paper a wide tree-like space is defined, and it is shown that the class of all wide tree-like spaces has the fixed point property for continuous functions. $A$ characterization of a wide tree-like space is revealed. This class of tree-like spaces contains many compact plane continua, all of which do not separate the plane. The same can be said about the class of tree-like spaces, but it is not known whether or not this class has the fixed point property for continuous functions.

1. Introduction. A bounded plane continuum which does not separate the plane can be represented as the intersection of the elements of a monotonic decreasing sequence of open 2 -cells. A well-known problem is to determine whether or not each such continuum has the fixed point property for continuous functions. This question has been answered in the affirmative for many special plane continua, e.g. [3]. The question of whether a tree-like space has the fixed point property for continuous functions was raised in a conversation with $\mathrm{O}$. H. Hamilton. It is the purpose of this paper to answer this question for wide tree-like spaces. The class of wide tree-like spaces contains many plane continua, all of which do not separate the plane. Likewise, the class of tree-like spaces contains many plane continua, all of which do not separate the plane.

We shall use Burgess's definition [2] of a linear chain and a definition similar to his of a tree-like chain, namely: a tree-like chain $C$ is a finite coherent collection of open sets such that (1) each two nonintersecting elements of $C$ are a positive distance apart; (2) no subcollection of $C$ consisting of more than two elements is a circular chain; and (3) no three elements have a point in common. If $C$ is a tree-like chain and $b \in C$, then $b$ is a branch link of $C$ if and only if there exists more than two other links of $C$ that intersect $b$. Also, if $l$ is a member of a tree-like chain $C$, then $l$ is called an end link of $C$ if and only if there exists only one other link of $C$ that intersects $l$. All spaces considered in this paper are metric spaces and $(M, d)$ denotes the metric space with set $M$ and metric $d$. 